\title{
Molecular Conformation and Packing of Poly(vinylidene fluoride). Stability of Three Crystalline Forms and the Effect of High Pressure
}

\author{
Ryozo Hasegawa*, Masamichi Kobayashi, and Hiroyuki Tadokoro \\ Department of Polymer Science, Faculty of Science, Osaka University, \\ Toyonaka, Osaka 560, Japan.
}

(Received January 6, 1972)

\begin{abstract}
The formation of three crystalline forms of poly(vinylidene fluoride) was studied in detail by using an apparatus for heat treatments under high pressure up to $5000 \mathrm{~atm}$. Form II consisting of $T G T \bar{G}$-type molecular chains was the most stable under atmospheric pressure, and form I, composed of planar zigzag-type chains, was formed under special conditions, such as tension, high pressure, etc. Form III may be an intermediate modification between I and II. Based upon these experimental facts, as well as the potential energy calculations of the intra- and intermolecular interactions in the crystal lattices due to the van der Waals and electrostatic forces, the relation between the conditions of formation of the three forms and their structures was examined. The planar zigzag-type conformation (in forms I and III) is considered to be less stable than the $T G T \bar{G}$ type (form II) because of the steric hindrances and electrostatic dipole interactions. In spite of the difference in the intramolecular potential energy between these two molecular conformations, the stabilities of the three crystalline forms are not so very different because of the more favorable intermolecular interaction in form I.

KEY WORDS Poly(vinylidene fluoride) / Molecular Conformation /

Molecular Packing / Crystal Form / High-Pressure Treatment /

Potential Energy Calculation / Van der Waals Interaction / Dipole

Interaction / Intramolecular Interaction / Intermolecular Interaction /
\end{abstract}

Many authors have studied the chemical and physical properties of poly(vinylidene fluoride) (PVDF). Kondrashov ${ }^{1}$ proposed two types of molecular form with different fiber identity periods of 2.55 and $4.66 \AA$ from the X-ray data of PVDF. Makarevich and Nikitin ${ }^{2}$ found that PVDF gave two kinds of infrared spectrum depending upon the preparation conditions. Gal'perin et al., ${ }^{3,4}$ and Natta, et al. ${ }^{5}$ confirmed the existence of two crystal forms designated as I and $\mathrm{II}^{* *}$ from the $X$-ray diffraction data.

Gal'perin, et al., ${ }^{3,4}$ suggested a planar zigzagtype conformation from the identity period $2.55 \AA$ for form I, and proposed the possibility of a

* Present address: Products Development Institute, Teijin Limited, Hinode-cho, Iwakuni, Yamaguchi, 740 Japan.

** Gal'perin, et al., ${ }^{3,4}$ and Okuda, et al., ${ }^{6}$ used the terms of $\beta$ and $\alpha$ forms for forms I and II, respectively.
$T G T \bar{G}$-type or a $(2 / 1)$ helical conformation from the identity period $4.57 \AA$ for form II. Here $T$, $G$, and $\bar{G}$ denote the trans and two types of gauche forms, respectively. Lando, et al., ${ }^{7,8}$ determined, by X-ray and wide-line NMR analyses, the crystal structure of form I with two planar zigzag chains in the orthorhombic unit cell. For form II, Doll and Lando ${ }^{9}$ proposed two possible crystal structures with space groups $P 2_{1}\left(C_{2}^{2}\right)$ and $P 1\left(C_{1}^{1}\right)$, each containing two chains of the conformation essentially of the $T G T \bar{G}$ type in the unit cell. Cortili and Zerbi ${ }^{10}$ suggested a planar zigzag type for form I and a $T G T \bar{G}$ type for form II on the basis of conformational analysis and vibrational spectroscopic considerations (factor group analysis). These two types of molecular structure were supported by the calculation of the normal vibrations by Enomoto, et al. ${ }^{11}$ Recent studies of Raman scattering by Boerio and Koenig ${ }^{12,13}$ supported the $T G T \bar{G}$ model of 
form II.

The existence of the third form was suggested at first by Natta, et al. ${ }^{5}$ in the specimen of a stretched molded sheet, and also suggested by Cortili and $\mathrm{Zerbi}^{14}$ in the film cast from a dimethyl sulfoxide solution. Their reports, however, were not conclusive. On the other hand, Doll and Lando $^{15}$ reported that crystallization of PVDF under high pressure yielded a new crystal form, and they had originally designated it as form III. Recently they suggested that the form crystallized under high pressure was actually a high-melting mixture of forms I and II. ${ }^{16}$ The authors reported in a previous paper ${ }^{17}$ that the pressure-crystallized form III was composed of planar zigzag chains and did not contain any detectable amount of form II. It will be confirmed in a subsequent paper ${ }^{18}$ that both the third form found in the film cast from dimethyl sulfoxide $^{6,14}$ and that found in the pressurecrystallized specimen have essentially the same crystal structure. Here this type of crystal structure is tentatively designated as form III.

The conditions of formation for the above three forms are complicated, and have been reported to be due to the kind of solvent, ${ }^{6,14}$ the content of head-to-head linkages, ${ }^{8,19}$ and hydrostatic pressure. ${ }^{15-17,19}$

The present work was undertaken to study more systematically the formation of the three types and the transitions among them under various conditions of heat treatment including those under high pressure. Moreover, in order to discuss the stability of three forms, the potential energy was calculated for the intra- and inter-molecular interactions in the crystal lattices due to the van der Waals and electrostatic forces. The crystal structures of three forms used here will be reported in ref 18 . Based upon the experimental facts as well as the results of the energy calculation, the authors wish to examine the relation between the conditions of formation of three types and their structures.

\section{EXPERIMNTAL}

\section{Preparation of Samples}

The sample used here was supplied by Kureha Chemical Industry Co., Ltd. The number-average molecular weight is 64000 , and the irregular content (head-to-head or tail-to-tail linkages) estimated by NMR measurement is less than $5 \%$. This sample always gave form II by melt-crystalization under atmospheric pressure. Films of type II were prepared by pressing the samples under $100-130 \mathrm{~kg} / \mathrm{cm}^{2}$ at $200-230^{\circ} \mathrm{C}$. Cast films and powder specimens were prepared from a solution in dimethyl sulfoxide or monochlorobenzene-dimethylformamide mixture of $90: 10$ volume ratio. ${ }^{6}$

\section{Heat Treatment under High Pressure}

The specimen (fiber or film) set on a metal holder was subjected to heat treatment under various temperatures and pressures. A highpressure apparatus, described in a previous paper, ${ }^{17}$ was used for the treatment under hydrostatic pressure at up to $5000 \mathrm{~atm}$. During long heat treatments $\left(e . g\right.$., for more than $12 \mathrm{hr}$ at $200^{\circ} \mathrm{C}$ ) the specimen became dark brown due to thermal degradation. An attempt at prevention was made by limiting the heating time to $1-6 \mathrm{hr}$ depending on the temperature.

\section{Characterization}

The specimens thus obtained were characterized by the following methods. Wide-angle X-ray diffraction patterns were obtained by using nickelfiltered $\mathrm{Cu}-\mathrm{K} \alpha$ radiation with a cylindrical camera (Rigakudenki Co., Ltd.). In order to obtain the precise cell dimensions, the spacings of the reflections were measured with reference to those of aluminum. Infrared spectra were taken with a Japan Spectroscopic Company DS-402G grating infrared spectrophotometer. The melting point was measured with a Perkin-Elmer DSC-1B differential scanning calorimeter, and also with a polarizing microscope. The density was measured by the flotation method with a carbon tetrachloride-ethylene bromide mixture as medium at $30^{\circ} \mathrm{C}$.

\section{FORMATION OF THREE MODIFICATIONS}

The conditions of formation and mutual transition of form I, II, and III are shown in Figure 1. All the transitions observed here are considered as occurring in the solid-state, because the shape and orietation of the specimen remained unaltered during the transition. The melting points and densities of the specimens crystallized 


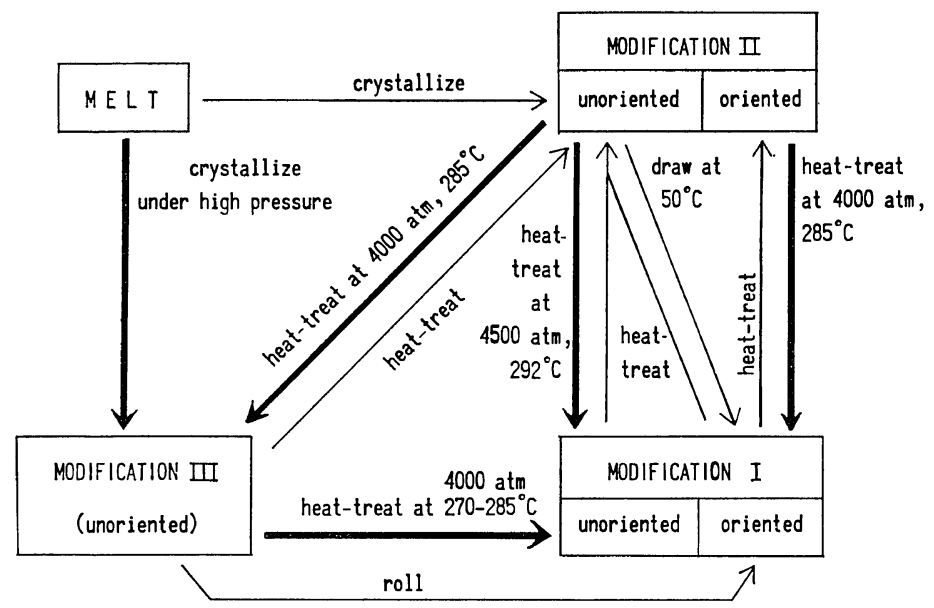

Figure 1. Conditions for transitions among three forms of poly(vinylidene fluoride): $(\rightarrow)$ crystallization or heat treatment under high pressure; $(\rightarrow)$ treatment under atmospheric pressure.

Table I. Melting points and densities of poly(vinylidene fluoride) crystallized in forms I, II, and III

\begin{tabular}{|c|c|c|c|}
\hline \multirow[b]{2}{*}{ Specimen } & \multirow{2}{*}{$\begin{array}{l}\text { Melting } \\
\text { point, } \\
{ }^{\circ} \mathrm{C}\end{array}$} & \multicolumn{2}{|c|}{ Density, g/cc } \\
\hline & & $\begin{array}{l}\text { Obsd } \\
\text { at } 30^{\circ} \mathrm{C}\end{array}$ & Calcd $^{\mathrm{a}}$ \\
\hline Form I & & & 1.97 \\
\hline Oriented specimen drawn at $50^{\circ} \mathrm{C}$ & 168 & $1.77_{1}$ & \\
\hline Oriented specimen heat-treated at $285^{\circ} \mathrm{C}, 4000 \mathrm{~atm}$ & 191 & $1.80_{2}$ & \\
\hline Unoriented specimen heat-treated at $285^{\circ} \mathrm{C}, 400 \mathrm{~atm}$ (from cast form III) & 173 & $1.80_{6}$ & \\
\hline Form II & & & 1.93 \\
\hline Melt-crystallized specimen & 175 & 1.769 & \\
\hline Oriented specimen drawn at $150^{\circ} \mathrm{C}$ & 178 & $1.78_{8}$ & \\
\hline $\begin{array}{l}\text { Specimen cast from a solution in a monochlorobenzene-dimethylform- } \\
\text { amide mixture }\end{array}$ & 176 & $1.80_{7}$ & \\
\hline Form III & & & 1.95 \\
\hline Melt-crystallized specimen at 4000 atm & 179 & $1.78_{4}$ & \\
\hline Unoriented specimen heat-treated at $285^{\circ} \mathrm{C}, 4000 \mathrm{~atm}$ (from form II) & 197 & $1.80_{2}$ & \\
\hline Specimen cast from a dimethyl sulfoxide solution & 171 & $1.80_{4}$ & \\
\hline
\end{tabular}

a Calculated from the unit cell listed in Table II.

in forms I, II, and III are listed in Table I. No appreciable differences in the melting point and density could be found among the three forms. The melting point and density of the specimens may depend on the crystallinity and also on the morphology. Crystallographic data of the three forms are summarized in Table II.

Form I. An oriented specimen of form I was obtained by drawing the melt-crystallized sample (unoriented form II) at $50^{\circ} \mathrm{C}$ (draw ratio $500 \%$ ). By heat treatment under high pressure $\left(285^{\circ} \mathrm{C}\right.$,
$4000 \mathrm{~atm}$ ), the oriedted specimen of form I did not show any transition to another form, but its crystallinity increased noticeably.

Form II. The unoriented specimen of form II was obtained by melt-crystallization under atmospheric pressure. The X-ray powder photograph of this specimen is shown in Figure 2(a). By drawing this specimen (draw ratio 400\%) at $150^{\circ} \mathrm{C}$, an oriented specimen of form II (containing small amount of form I) was obtained. As Okuda, et al., reported, ${ }^{6}$ form II was also 
Table II. Crystallographic data of three forms of poly(vinylidene fluoride)

\begin{tabular}{|c|c|c|c|}
\hline & Form I & Form II & Form III \\
\hline Crystal system & orthorhombic & monoclinic & monoclinic \\
\hline Space group & $\mathrm{Cm} 2 \mathrm{~m}\left(\mathrm{C}_{2 \mathrm{~V}}^{14}\right)$ & $\mathbf{P} 2_{1} / \mathbf{c}\left(\mathbf{C}_{2 \mathrm{~h}}^{5}\right)$ & $\mathrm{Cl} 21\left(\mathrm{C}_{2}^{3}\right)$ \\
\hline \multirow[t]{4}{*}{ Lattice constants } & $a=8.58 \AA$ & $a=4.96 \AA$ & $a=8.66 \AA$ \\
\hline & $b=4.91 \AA$ & $b=9.64 \AA$ & $b=4.93 \AA$ \\
\hline & $c(\text { f.a. })^{a}=2.56 \AA$ & $c$ (f.a. $)=4.62 \AA$ & $c($ f.a. $)=2.58 \AA$ \\
\hline & & $\beta=90^{\circ}$ & $\beta=97^{\circ}$ \\
\hline Number of chains per lattice & 2 & 2 & 2 \\
\hline Molecular conformation ${ }^{\mathrm{b}}$ & $T_{2}$ & $T G T \bar{G}$ & $T_{2}$ \\
\hline Density, Obsd at $30^{\circ} \mathrm{C}$ & $1.80_{6} \mathrm{~g} / \mathrm{cc}$ & $1.76_{9} \mathrm{~g} / \mathrm{cc}$ & $1.80_{4} \mathrm{~g} / \mathrm{cc}$ \\
\hline Calcd (X-ray) & $1.97 \mathrm{~g} / \mathrm{cc}$ & $1.93 \mathrm{~g} / \mathrm{cc}$ & $1.94 \mathrm{~g} / \mathrm{cc}$ \\
\hline
\end{tabular}

a f.a., fiber axis.

b $T, G$, and $\bar{G}$ denote the trans and two types of gauche forms, respectively.

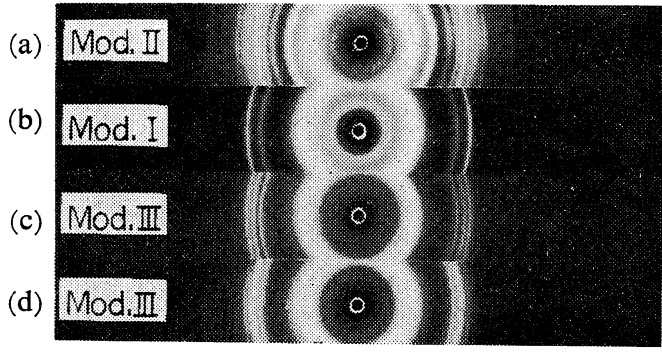

Figure 2. X-ray powder patterns of poly(vinylidene fluoride): (a) form II in the melt-crystallzed specimen; (b) form I obtained by heat treatment of the cast specimen (form III); (c) form III obtained by heat treatment of the melt-crystallized sample (form II) at $285^{\circ} \mathrm{C}, 4000 \mathrm{~atm}$; (d) form III in the specimen cast from a dimethyl sulfoxide solution.

found in the precipitate from a solution in monochlorobenzene-dimethylformamide mixture or an acetone solution.

Form III. Under a pressure of $4000 \mathrm{~atm}$, form III was obtained by melt-crystallization and also by heat treatment of unoriented films of form II at $285^{\circ} \mathrm{C}^{17}$ As Cortili and Zerbi reported, ${ }^{15}$ a dimethyl sulfoxide solution yielded a precipitate of form III, which has been reported as the $\beta$ form by Okuda, et al. ${ }^{6}$ The X-ray patterns of the specimen subjected to heat treatment under high pressure (containing none of form II) and of that cast from dimethyl sulfoxide are shown in Figures 2(c) and (d), respectively. In spite of the slight difference in the intensity, all the reflections from both of the specimens were indexed by the same unit cell proposed in ref 18 .

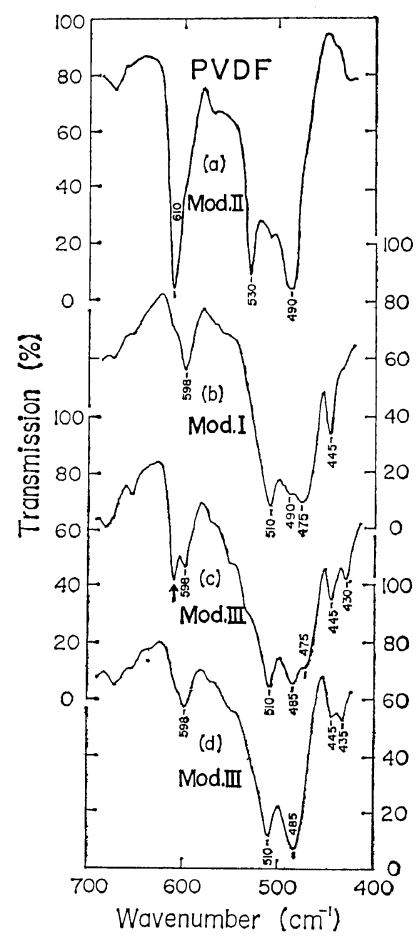

Figure 3. Infrared spectra of poly(vinylidene fluoride); (a) form II; (b) form I; (c) form III obtained by heat treatment under high pressure, containing small amount of form II as shown by an arrow $(\uparrow$ ); (d) form III cast from dimethyl sulfoxide. Spectra of (a), (b), and (d) correspond to the X-ray patterns of Figures 2(a), (b), and (d), respectively.

Both the specimens are therefore denoted as form III. Although Doll and Lando ${ }^{16}$ suggested no third crystal form, this result indicated the exist- 
ence of it. The infrared spectra of the above two specimens of form III are shown in Figures $3(\mathrm{c})$ and (d), respectively, in comparison with those of form II(a) and form I(b). Perfect transformation into form III of thin films (ca. $30 \mu)$ suitable for infrared measurement was not possible because of the thermal degradation, and so the spectrum of Figure 3(c) contains small fraction of form II whose band is indicated by an arrow. The similar infrared spectra of forms I and III suggest a planar zigzag molecular structure in form III. $^{17}$

The specimen obtained from a dimethyl sulfoxide solution may be the pure form III, since the specimen prepared under high pressure gave the X-ray patterns and infrared spectra having the feature of the superposition of form $I$ and of the form III cast from a dimethyl sulfoxide solution.

\section{Transition under High Pressure}

The several types of transition caused by heat treatment under high pressure are shown by thick arrows in Figure 1. In these transitions, the formation of type III by melt-crystallization under high pressure was found at first by Doll and Lando $^{15}$ (thereafter they denied form III as mentioned above $\left.^{16}\right)$, and the others were found by the authors. ${ }^{17}$

As mentioned above, form III was obtained by heat treatment of the unoriented form II under $4000 \mathrm{~atm}$ at $285^{\circ} \mathrm{C}$. On the other hand, unoriented form I was obtained from the same unoriented form II under a higher pressure (4500 atm) at $292^{\circ} \mathrm{C}$. By heat treatment under 4000 atm at $270-285^{\circ} \mathrm{C}$, form III cast from a dimethyl sulfoxide solution was transformed into form I. The same transformation was not detected in the case of the pressure-crystallized form III owing to the thermal degradation of the specimen. The $\mathrm{X}$-ray pattern and infrared spectrum of the form $I$ thus obtained are shown in Figures $2(b)$ and 3(b), respectively. Although unoriented form I had already been obtained using a copolymer of vinylidene fluoride and tetrafluoroethylene by Lando and Doll, ${ }^{8}$ unoriented form I of PVDF homopolymer can be obtained by the above treatment.

In the case of the precipitate from a solution in monochlorobenzene-dimethylformamide mix- ture ${ }^{6}$ form II was transformed directly into form I by heat treatment at $281-285^{\circ} \mathrm{C}$ under 4000 atm. This fact differs from the case of the meltcrystrllized form II mentioned above. One of the reason for this may be that the precipitate has a different morphology $y^{6,20}$ and reacts to pressure treatment in a different way from the melt-crystallized specimen.

By rolling the film, form III transformed into oriented form I. In an attempt to obtain an oriented specimen of form III, heat treatment of the oriented form II was carried out at $285^{\circ} \mathrm{C}$ under $4000 \mathrm{~atm}$. However, an oriented specimen of form I was obtained by this treatment. ${ }^{17}$ So far oriented form III has not been obtained.

\section{Transitions under Atmospheric Pressure}

Form II was produced by heat treatment of the oriented specimen of form I at $185^{\circ} \mathrm{C}$ under tension as reported by Lando and $\mathrm{Doll}^{8}$ (see Figure 1). The orientation of the specimen almost disappeared after more than $1 \mathrm{~min}$, of the treatment while it was retained by a shorter treatment. Heat treatment of the unoriented specimens of forms I and III at $175^{\circ} \mathrm{C}$ produced unoriented form II in the specimen.

\section{ENERGY CALCULATION}

\section{Assumptions}

In the present calculation, the van der Waals interaction (V) and the electrostatic interaction (U) were considered. For the van der Waals interactions between all pairs of nonbonded atoms, the Lennard-Jones 6-12 potential function of the following form was used;

$$
V(r)=\varepsilon\left[\left(r_{\mathrm{min}} / r\right)^{12}-2\left(r_{\mathrm{min}} / r\right)^{6}\right],
$$

where $V(r)$ is the potential energy of two atoms at a distance $r, \varepsilon$ the depth of the potential energy minimum, and $r_{\mathrm{min}}$ the position of the minimum potential. For a pair of atoms of different kinds, $a$ and $b$, an empirical combining $\mathrm{law}^{21}$ may be used to estimate $r_{\min }(\mathrm{ab})$ and $\varepsilon(\mathrm{ab})$,

$$
\left.\begin{array}{l}
r_{\min }(\mathrm{ab})=\frac{1}{2}\left[r_{\min }(\mathrm{aa})+r_{\min }(\mathrm{bb})\right], \\
\varepsilon(\mathrm{ab})=\sqrt{\varepsilon(\mathrm{aa}) \varepsilon(\mathrm{bb})} .
\end{array}\right\}
$$

The values of interaction parameters, $r_{\min }$ and $\varepsilon$, for carbon, ${ }^{21}$ fluorine, ${ }^{22,23}$ and hydrogen atoms ${ }^{24}$ are listed in Table III. Here, the interaction 
Table III. Lennard-Jones 6-12 interaction parameters

\begin{tabular}{lccc}
\hline $\begin{array}{c}\text { Nonbonded } \\
\text { atom }\end{array}$ & $r_{\min }, \AA \begin{array}{c}\varepsilon, \mathrm{kcal} / \mathrm{mol} \\
\text { of pair }\end{array}$ & Ref \\
\hline Carbon & 3.40 & 0.1280 & $\begin{array}{c}\text { McCullough and } \\
\text { McMahon }\end{array}$ \\
Fluorine & 3.16 & 0.0718 & $\begin{array}{c}\text { Mason and } \\
\text { Rice } 22,23\end{array}$ \\
Hydrogen & 2.40 & 0.1222 & $\begin{array}{c}\text { Scott and } \\
\text { Scheraga }\end{array}$ \\
\hline
\end{tabular}

between two fluorine atoms was assumed to be the same as those between two isolated neon atoms after Mason and Kreevoy. ${ }^{23}$

The electrostatic interactions between the polar $\mathrm{CF}_{2}$ groups were calculated according to the dipole-dipole interaction equation;

$$
U_{i j}=\left[\left(\boldsymbol{\mu}_{i} \cdot \boldsymbol{\mu}_{j}\right)-3\left(\boldsymbol{\mu}_{i} \cdot \boldsymbol{r}_{i j}\right)\left(\boldsymbol{\mu}_{j} \cdot \boldsymbol{r}_{i j}\right) / r_{i j}^{2}\right] /\left(D r_{i j}^{3}\right),
$$

where $U_{i j}$ is the interaction energy between the $i$-th and $j$-th point dipoles, $\mu_{i}$ and $\mu_{j}, r_{i j}$ the vector directing from the $i$-th to the $j$-th point dipole, and $D$ the dielectric constant. A point dipole was assumed to be located at the midpoint of the line connecting the midpoints of the two $\mathrm{C}-\mathrm{F}$ bonds in a $\mathrm{CF}_{2}$ group. The value of the $\mathrm{C}-\mathrm{F}$ bond moment was taken as 1.41 Debye. $^{25}$ The dielectric constant was assumed here to be $4.0 .^{24}$

If one considers a crystal consisting of $M$ polymer molecules, $M$ being infinitely large. The total interaction energy $E$ of the whole crystal can be written by the following equation

$$
E=M E_{\mathrm{intra}}+\sum_{m=1}^{M-1} \sum_{m^{\prime}>m}^{M} E\left(m, m^{\prime}\right),
$$

where $E_{\text {intra }}$ is the intramolecular interaction energy of a polymer molecule, and $E\left(m, m^{\prime}\right)$ is the intermolecular interaction energy between the $m$-th and $m^{\prime}$-th polymer molecules. Assuming a polymer molecule which consists of $2 N+1$ crystallographic asymmetric units $(-N,-N+1$, $\ldots,-1,0,1, \ldots, N-1, N)$ and satisfies the Born's cyclic boundary condition, $E\left(m, m^{\prime}\right)$ is written in the form

$$
E\left(m, m^{\prime}\right)=\sum_{i=-N}^{N} \sum_{j=-N}^{N} E\left(m_{i} m_{j}^{\prime}\right),
$$

where $E\left(m_{i}, m_{j}{ }^{\prime}\right)$ is the interaction energy between the $i$-th unit in the $m$-th molecule and the $j$-th unit in the $m^{\prime}$-th molecule. The intramolecular potential energy per unit $E_{\text {intra }}$ (u.) is written by the equation;

$$
\begin{aligned}
E_{\text {intra }}(\text { u. }) & =\frac{E_{\text {intra }}}{2 N+1} \\
& =\frac{1}{2 N+1}\left[\frac{1}{2} \sum_{i=-N}^{N} \sum_{j=-N}^{N} E(i, j)\right] \\
& =\frac{1}{2} \sum_{k=-N}^{N} E(0, k)
\end{aligned}
$$

where $E(i, j)$ is the interaction energy between the $i$-th and $j$-th units in a molecule for $i \neq j$. Here $m$ or $m^{\prime}$ is omitted for simplicity, since $m=m^{\prime}$. The interaction energy within the $i$-th unit is given by $\frac{1}{2} E(i, i)$. It is assumed here that $E(0, k)$ is composed of the van der Waals energy $V(0, k)$ and the electrostatic energy $U(0, k)$;

$$
\begin{aligned}
E(0, k) & =V(0, k)+U(0, k) \\
& =\sum_{p=1}^{n} \sum_{q=1}^{n} V\left(0_{p}, k_{q}\right)+\sum_{r=1}^{t} \sum_{s=1}^{t} U\left(0_{r}, k_{s}\right),
\end{aligned}
$$

where $V\left(0_{p}, k_{q}\right)$ [or $\left.U\left(0_{r}, k_{s}\right)\right]$ is the van der Waals energy (or the electrostatic energy) between the $p$-th atom (or the $r$-th point dipole) in the 0 -th unit and the $q$-th atom (or the $s$-th point dipole) in the $k$-th unit, and $n$ and $t$ are the numbers of atoms and point dipoles involved in an asymmetric unit, respectively. In $E(0,0)$ the terms with $p=q$ or $r=s$ vanish. In the case of PVDF, $n=6$ and $t=1$.

The intermolecular interaction energy per unit $E_{\text {inter }}(\mathbf{u}$.$) is written in the form$

$$
\begin{aligned}
E_{\text {inter }}(\text { u. })= & \frac{1}{M(2 N+1)} \sum_{m=1}^{M-1} \sum_{m^{\prime}>m}^{M-1} E\left(m, m^{\prime}\right) \\
= & \frac{1}{M(2 N+1)} \sum_{m=1}^{M-1} \sum_{m^{\prime}>m}^{M} \sum_{i=-N}^{N} \sum_{j=-N}^{N} \\
& \times E\left(m_{i}, m_{j}^{\prime}\right) .
\end{aligned}
$$

When an assymetric unit consists of two or more monomeric units, $E($ u.) should be divided by the number of monomeric units involved. In the present case, an assymetric unit contains one monometric unit.

Actually the calculation was made by utilizing the symmetry of the crystal lattice. The energy terms which do not depend on the conformation were omitted in the intramolecular energy calculation. The calculations covered all the atom pairs with the distances shorter than $10 \AA$ for the 
van der Waals interactions, and the point dipole pairs of the distances shorter than $50 \AA$. The dipole interaction energy was found to converge within $1 \%$ at $30 \AA$. The calculations were performed by using a NEAC 2200 Model 500 digital computer installed in the authors' university.

\section{Results of Calculation}

The results are given in Table IV for forms I and II. The values given in parentheses are the components of intra- and intermolecular interactions; the van der Waals and the electrostatic interactions. For form I the calculation was made on the planar zigzag structure for simplicity, although the X-ray crystal analysis suggested a statistically disordered structure with alternatelydeflected zigzag chains. ${ }^{18}$ The energy calculation of form II was made on the structure which will be reported in ref 18 . The interaction energy for form III is not shown, since the accuracy of the lattice constants and atomic parameters of form III is lower than those of the other two forms.

Table IV. Intra- and Intermolecular potential energy of forms I and II ( $\mathrm{kcal} / \mathrm{mol}$ monomeric unit)

\begin{tabular}{ccc}
\hline Form & I $(T T)$ & II $(T G T \bar{G})$ \\
\hline Intramolecular interaction & -0.48 & -1.46 \\
(Van der Waals) & $(-1.19)$ & $(-1.57)$ \\
(Electrostatic) & $(+0.71)$ & $(+0.11)$ \\
Intermolecular interaction & -5.25 & -4.57 \\
(Van der Waals) & $(-5.06)$ & $(-4.44)$ \\
(Electrostatic) & $(-0.19)$ & $(-0.13)$ \\
Total & -5.73 & -6.03 \\
\hline
\end{tabular}

In the case of form I, the intramolecular interaction energy was also calculated for the alternately-deflected zigzag chain corresponding to the disordered crystal structure which will be reported in ref 18 . For the molecular chain illustrated at the top of Figure 4, the deflection angle $\sigma$ was defined as the deviation of the main chain from the original zigzag plane. As shown in Figure 4, the intramolecular potential energy was calculated for various deflection angles from $0^{\circ}$ to $15^{\circ}$. The angle of about $7^{\circ}$ gives a minimum value $-0.56 \mathrm{kcal} / \mathrm{mol}$ monomeric unit, just coinciding with the angle giving the minimum discrepancy factor $R$ in the X-ray analysis (ref

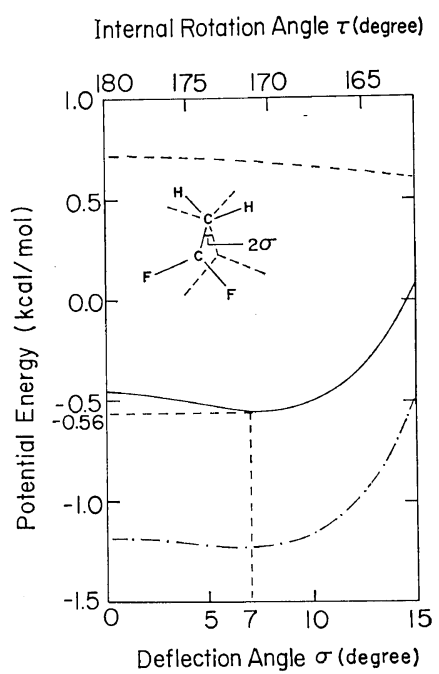

Figure 4. Variation of intramolecular potential energy of the alternately-deflected zigzag chain with the deflection angle $\sigma$ : (-) total potential energy; (--) van der Waals energy; (---) electrostatic energy.

18). This result suggests the possibility of the alternately-deflected zigzag chain in the crystal lattices of forms I and III. This deflected zigzag chain has the internal rotation angle of the main chain of $172^{\circ}$, which is comparable with that of poly(tetrafluoroethylene) with the $(13 / 6)$ helical conformation $\left(165^{\circ}\right){ }^{26,27}$

\section{DISCUSSION}

As shown in Figure 5 , the $\mathrm{F}$...F distance is $2.56 \AA$ in form I, and $2.60 \AA$ even if the alternatelydeflected structure is assumed, whereas the

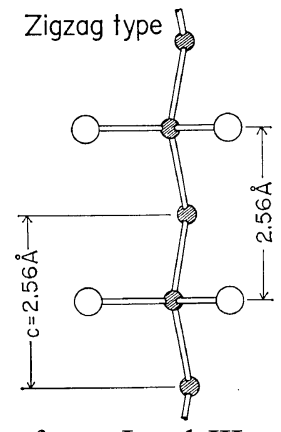

forms I and III

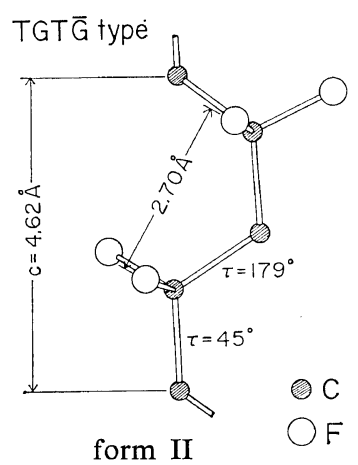

form II
Figure 5. Molecular structures of form I (TT type) and form II ( $T G T \bar{G}$ type). 
distance is $2.70 \AA$ in form II just coinciding with twice the van der Waals radius of the fluorine atom (described in detail in ref 18). From this steric hindrance and the dipole interactions between $\mathrm{CF}_{2}$ groups, the planar zigzag-type conformation is supposed to be less stable than the $T G T \bar{G}$ type. Such a situation was actually shown by the potential energy calculation in Table IV; the intramolecular interaction energy of the planar zigzag type (form I) is $-0.48 \mathrm{kcal} / \mathrm{mol}$ monomeric unit, much higher than the value of the $T G T \bar{G}$ type (form II), $-1.46 \mathrm{kcal}$. This may explain the experimental facts that form II is stable under ordinary condition, and that the formation of types I and III requires the special conditions mentioned above. The higher energy value of the van der Waals interaction $(-1.19 \mathrm{kcal} / \mathrm{mol}$ monomeric unit) and the electrostatic interaction energy $(+0.71 \mathrm{kcal} / \mathrm{mol}$ monomeric unit) of form I may be due to the steric hindrance between the neighboring $\mathrm{CF}_{2}$ groups and the parallel array of the $\mathrm{CF}_{2}$ dipole moments, respectively.

In spite of the large difference between the intramolecular potential energies of the planar zigzag- and $T G T \bar{G}$-type conformations, the stabilities of the crystals of forms I and II are not very different, viz., -5.73 and $-6.03 \mathrm{kcal} / \mathrm{mol}$ monomeric unit. This may be due to the intermolecular interactions in the crystalline lattices. The intermolecular interaction energy of form I is $-5.25 \mathrm{kcal} / \mathrm{mol}$ monomeric unit, appreciably lower than that of form II, $-4.57 \mathrm{kcal}$. This result may originate from the more favorable intermolecular interactions; the more compact packing and the lower electrostatic energy in form I. This may cover the high energy of intramolecular interactions of the planar zigzag conformation if the aforementioned conditions are satisfied.

Based on the accurately measured diffraction spacings, the crystalline densities were estimated as $1.97 \mathrm{~g} / \mathrm{cc}$ for form I, $1.94 \mathrm{~g} / \mathrm{cc}$ for form III, and $1.93 \mathrm{~g} / \mathrm{cc}$ for form II. ${ }^{18}$ The effect of high pressure on the stabilities of three forms, ${ }^{17}$ that is, the stability in the order of forms I, III, and II, may be closely related to the order of these density values.

The molecular orientation may also be closely related to the polymorphism of PVDF. Oriented form III has not yet been obtained either by rolling the unoriented form III or by heat treatment of the oriented form II under high pressure.

Acknowledgement. The authors wish to express their appreciation to Kureha Chemical Industry Co., Ltd. for supplying the sample of poly(vinylidene fluoride), and also to Mr. K. Tai of this laboratory for his collaboration in energy calculations.

\section{REFERENCES}

1. Yu. D. Kondrashov, Tr. GIPKh'a, No. 46, 166 (1960).

2. N. I. Makarevich and V. N. Nikitin, Vysokomol. Soedin., 7, 1673 (1965).

3. Ye. L. Gal'perin, Yu. V. Strogalin, and M.P. Mlenik, ibid., 7, 933 (1965).

4. Ye. L. Gal'perin and B.P. Kosmynin, ibid., 11, 1432 (1969).

5. G. Natta, G. Allegra, I. W. Bassi, D. Sianesi, G. Caporiccio, and E. Torti, J. Polym. Sci., Part A, 3, 4263 (1965).

6. K. Okuda, T. Yoshida, M. Sugita, and M. Asahina, ibid., Part B, 5, 465 (1967).

7. J. B. Lando, H. G. Olf, and A. Peterlin, $J$. Polym. Sci., Part A-1, 4, 941 (1966).

8. J. B. Lando and W. W. Doll, J. Macromol. Sci., B2, 205 (1968).

9. W.W. Doll and J.B. Lando, ibid., B4, 309 (1970).

10. G. Cortili and G. Zerbi, Spectrochim. Acta, 23A, 285 (1967).

11. S. Enomoto, Y. Kawai, and M. Sugita, $J$. Polym. Sci., Part A-2, 6, 861 (1968).

12. F. J. Boerio and J. L. Koenig, ibid., 7, 1489 (1968).

13. F. J. Boerio and J. L. Koenig, ibid., 9, 1517 (1971).

14. G. Cortili and G. Zerbi, Spectrochim. Acta, 23A, 2216 (1967).

15. W. W. Doll and J. B. Lando, J. Macromol. Sci., B2, 219 (1968).

16. W.W. Doll and J.B. Lando, ibid., B4, 889 (1970).

17. R. Hasegawa, Y. Tanabe, M. Kobayashi, H. Tadokoro, A. Sawaoka, and N. Kawai, $J$. Polym. Sci., Part A-2, 8, 1073 (1970).

18. R. Hasegawa, Y. Takahashi, Y. Chatani, and H. Tadokoro, Polymer J., 3, 600 (1972).

19. W. W. Doll ane J. B. Lando, J. Macromol. Sci., B4, 897 (1970).

20. K. Sakaoku and A. Peterlin, ibid., B1, 401 (1967).

21. R. L. McCullough and P. E. McMahon, Trans. Faraday Soc., 60, 2089 (1964). 
Conformation and Packing of Poly(vinylidene fluoride)

22. E. A. Mason and W. E. Rice, J. Chem. Phys., 22, 843 (1954).

23. E. A. Mason and M. M. Kreevoy, J. Amer. Chem. Soc., 77, 5808 (1955).

24. R. A. Scott and H. A. Scherage, J. Chem. Phys., 45, 2091 (1966).

25. C. P. Symth, "Dielectric Behavior and Struc- ture,' McGraw-Hill, New York, N.Y., p 1955, 244.

26. C. W. Bunn and E. R. Howells, Nature, 174, 549 (1954).

27. P. De Santis, E. Giglio, A. M. Liquori, and A. Ripamonti, J. Polym. Sci., Part A, 1, 1383 (1963). 